\title{
Lithium intoxication in infectious context of a patient on chronic Lithium therapy
}

\author{
Mihaela Zaharia ${ }^{1}$, Augustin Cupsa ${ }^{1}$, \\ Corneliu P. Popescu ${ }^{1,2}$, Ionut Vlad ${ }^{3}$, Simin Florescul, ${ }^{1,2}$, Emanoil Ceausu ${ }^{1}$ \\ 1"Dr. Victor Babes" Clinical Hospital of Infectious and Tropical Diseases, Bucharest, Romania \\ 2"Carol Davila" University of Medicine and Pharmacy, Bucharest, Romania \\ ${ }^{3}$ Faculty of General Medicine, Oradea, Romania
}

\begin{abstract}
Introduction. In the history of his usage, by the 1850`s, Lithium was first used for the treatment of the gout. As mania and melancholia were included in the same diagnostic group, Lithium started to be used for the treatment of mental disorders. Nowadays, Lithium is still used in the bipolar disorder and as adjuvant in de-pression, schizophrenia, schizoaffective disorder and for the control of child aggressiveness. Methods and objective. Reporting a case of Lithium Intoxication in a patient with infection and chronic treat-ment with Lithium Carbonate.

Objectives. Reporting a case of Lithium Intoxication in a patient with streptococcal angina infection and chronic treatment with Lithium Carbonate.

Conclusion. Lithium intoxication may be rare in medical practice because of rare usage of Lithium therapy and strict monitorization of serum concentration. In this case, the tonsillar infectious accompanied by fever, impossibility of proper oral hydration and impairment of renal functions were the initial trigger of the pathologi-cal mechanism of intoxication syndrome. Secondary, the excretion of Lithium decreased and the low serum volume led to high blood concentration of Lithium. Later on, the gastrointestinal impairment which appeared slowly added to the vicious circle which involved hydration, excretion and serum concentration of Lithium producing and intoxication syndrome.
\end{abstract}

Keywords: infection, streptococcal angina, Lithium, intoxication, neurologic manifestations

\section{INTRODUCTION}

In the history of his usage, by the 1850`s, Lithium was first used for the treatment of the gout. As mania and melancholia were included in the same diagnostic group, Lithium started to be used for the treatment of mental disorders. In the 1920 `s, the psychiatrist H.J. Schou states the inefficiency of Lithium in the prevention of depression and therefore, Lithium therapy is temporary put aside. Starting with the 50`s Lithium is again used as a first line agent in affective disorders but is once again abandoned in 1977 after some studies put into light its nephro-toxic effects (1-3). Nowadays, Lithium is still used in the bipolar disorder and as adjuvant in depression, schizophrenia, schizoaffective disorder and for the control of child aggressiveness (1-4). In Romania, Lithium therapy is rarely used even if Lithium-based medicines are no longer produced, nor available in pharmacies.

\section{CLINICAL REPORT}

Male aged 48 has sore throat and dysphagia with a fever for 7 days, with insidious onset of intentional tremor of the limbs (with upper limb predominance) which progressively worsened 
to myoclonus, ataxia and mild dysarthria. In the last 4 days before admission into the ER unit, he had nausea and 3 vomiting food episodes with impairment of oral hydration during the previous 24 hours to admission.

The medical history of the patient shows depressive episodes with psychotic features and the absence of neurological history. Starting with 2012, the patient was constantly in treatment with Venlafaxine (225 mg/day) and Lithium Carbonate. Last dosage of lithium levels (3 weeks prior to hospitalization) was $1.3 \mathrm{mEq} / \mathrm{L}$ (normal range $=0.7-1.2 \mathrm{mEq} / \mathrm{l}$ ) and therefore he had his daily dose reduced to $450 \mathrm{mg} \mathrm{x} \mathrm{3/day.} \mathrm{In}$ the last 48 hours, the psychiatric medication was completely stopped by the patient because of digestive distress.

Clinical assessment reveals - fever of 38.1C, anxiety, but clear consciousness with proper orientation in time and space, limbs myoclonus with upper limbs predominance exacerbated by movement, positive tests for dysmetria, ataxia, no sensory or motor deficit, mild dysarthria without cranial nerve deficit, plantar coetaneous reflex in flexion, osteotendinous reflexes present, without signs of meningeal irritation, dehydrated tongue, pharyngeal and tonsils hyperemia, with tonsils hypertrophy and pus deposits in the tonsils, cardio-vascular and respiratory stability (BP $=120 / 80 \mathrm{mmHg}, \mathrm{HR}=80$ / min regular), slight bradycardia in febrile context.

Laboratory tests reveal - leukocytosis $(23,000 / \mathrm{microL})$ with neutrophilia, inflammatory syndrome increased hematocrit, hyponatremia $(\mathrm{Na}=126 \mathrm{mmol} / \mathrm{l})$, hypokalemia $(\mathrm{K}=2.8$ $\mathrm{mmol} / 1)$, nitrogen retention, creatinine $=1.7$ $\mathrm{mg} / \mathrm{dl}$ (normal range $=0.7-1.1 \mathrm{mg} / \mathrm{dl}$ ), normal blood sugar, cerebrospinal fluid is normal, native brain CT without signs of intracranial hypertension, HIV serology, TPHA/VDRL, Borrelia, herpes simplex 1,2, Chlamydia and Mycoplasma are negative, throat swabs positive for beta hemolytic streptococcus group $\mathrm{C}$, blood cultures remained unleavened 7 days. Plasmatic Lithium levels performed at 2 days after admission and (4 days after stopping administration of Lithium Carbonate) stands out a level of $1.4 \mathrm{mEq} / \mathrm{l}$ (normal range between 0.6-1.2 mEq/L). Electrocardiogram (ECG) performed at admission: sinus rhythm decreased of $\mathrm{T}$ wave amplitude, with mild ST-segment depression of $0.5 \mathrm{~mm}$, without U wave, with normal QT interval.

Taking into consideration the medical history and the clinical presentation at admission, the suspicion of lithium intoxication is raised. The electrolytes balance checked after admission and the lithium plasma levels are subsequent arguments for the diagnosis. The onset of streptococcal angina led to fever and secondary dehydration, vomiting and low fluid intake (sore throat and dysphagia). The treatment begun immediately with parenteral hydration and rebalancing electrolyte and antibiotic treatment with amoxicillin + clavulanic acid 1.2 g i.v. every 12 hours.

Later on, the neurological and psychiatric evaluation confirms the diagnosis with medical treatment recommendation: Clonazepam 0.5 mgx 2/day, Valproic Acid 500 mg/day and Venlafaxine (only) $75 \mathrm{mg} /$ day. The diagnostic of meningitis (unlikely in the clinical context) was excluded on the grounds of spinal tap examination which showed normal glucose and protein levels in cerebrospinal spinal fluid and normal cells.

Following this treatment, the patient's clinical evolution is favorable, with apyrexia in the next 48 hours after the initiation of antibiotic treatment and disappearance of throat symptoms, normalization of electrolyte balance and renal function and gradual remission of neurological manifestations in 7 days, when discharge is decided.

\section{DISCUSSIONS}

There are few case reports in the scientific literature for Lithium intoxication/overdose and 
infectious diseases - one case of enterocolitis (5) and Lithium intoxication reported in 2006 and one case of pneumonia and Lithium intoxication with secondary cerebellum atrophy (and unremitted ataxia) (6). There is also one case of Lithium intoxication for patient while treated simultaneously with Acyclovir for Herpes Zoster7 but there are many reported cases for Lithium overdose and drugs which inhibits renal function and excretion and also insufficient hydric ingestion. (8)

The very first signs for Lithium intoxication are the neurological signs shown by the tremble of the upper limbs, ataxia and dysarthria (the cerebellum toxic impact of Lithium) (9) and gastrointestinal symptoms such as nausea, vomiting and diarrhea. All of these signs where present within the clinical aspect of our patient. Also, due to hydro-electrolytic misbalance produced by Lithium during the intoxication, low serum $\mathrm{K}+$ may appear leading to cardiac arrhythmia $(1,4)$. We had for our patient a serum concentration of $\mathrm{K}$ initially of $2.8 \mathrm{mEq} / \mathrm{L}$ which was quickly raised to the normal value by intravenous administration of Potassium avoiding therefore any cardiac incidents for this patient.

Lithium has an initial plasma half-time of 1.3 days after first administration which eventually become 2.4 days after chronic administration (more than 1 year) and this prolonged half-time may lead to a cumulative effect $(1,4)$. On the other hand, the blood-brain barrier permits only a slow passage of the Lithium ions and that is why the intoxication with Lithium also has a slow remission. Lithium is solely excreted through urine and suffers no hepatic metabolism $(1,3,4)$. Serum concentration should be rigorously followed during Lithium therapy. The therapeutic values for Lithium range generally between 0,5 and $1.2 \mathrm{mEq} / \mathrm{L}$ (3). Our patient had initially a value of $1.4 \mathrm{mEq} / \mathrm{L}$ and that 4 days after ceasing his Lithium medication.

A mild Lithium intoxication is produced when the Lithium serum goes between 1.4-2.0 mEq/L and presents signs and symptoms such as - nausea, vomiting, abdominal pains, ataxia, hands shaking, mental incoherence, confusion, hypotonia, psychomotor agitation or retardation.

Serum concentrations of 2.0 up to $2.5 \mathrm{mEq} / \mathrm{L}$ may result in fasciculation, seizures, delusions, delirium and coma while concentration above $2.5 \mathrm{mEq} / \mathrm{L}$ leads to a severe intoxication episode which may be lethal. (4).

Our patient's clinical aspect comprised symptoms relevant for both mild and medium intoxication syndrome probably explained by higher Lithium concentration at the onset of the syndrome which remained unknown due to the delay of Lithium plasma concentration dosage.

Lithium intoxication is a medical emergency (4) and treatment must start immediately the clinical suspicion is raised. The first step is to immediately cut Lithium administration and rebalance the patient with hydric and electrolytic support (10).

Clinical evaluation should include neurologic, psychiatric and cardiologic assessments while the biologic screening should include Lithium and other electrolytes serum dosage, ECG, and renal function evaluation (11). In case of severe acute intoxication, one may initiate gastric wash ups, forced emesis and administration of polystyrene sulfonate. (4) In case of concentrations above $4 \mathrm{mEg} / \mathrm{L}$ blood or peritoneal dialysis may be necessary (10).

The risk of Lithium intoxication is directly related to high dosage, dehydration and low Sodium diet $(4,10,12)$.

In this case, the streptococcal angin led to fever and swallow impairment which further on led to low hydric ingestion and increased catabolism creating favorable condition for high levels of plasmatic Lithium level (though oral medication with Lithium was reduced and even stopped). In a second stage, the nausea, vomiting and limbs shaking worsen the dehydration and increased further more the toxic levels of Lithium producing a vicious circle which was fi- 
nally broken by the therapeutic intervention, mainly the intravenous hydro-electrolytes rebalance and intiation of antibiotics.

\section{CONCLUSIONS}

Lithium intoxication may be rare in medical practice because of rare usage of Lithium therapy and strict monitorization of serum concentration. In this case, the tonsillar infectious accompanied by fever, impossibility of proper oral hydration and impairment of renal functions were the initial trigger of the pathological mech-

\section{REFERENCES}

1. Maudsley. Therapheutic prescribing guide in Psychiatry, 7th Edt, Dunitz, 2003; Lithium: 96-103

2. Peet M., Pratt J.P. Lithium. Current status in Psychiatric Disorders. Drugs 1993; 46:7-17

3. Post, R.M. In Gelders, Andreasen (eds) New Oxford Textbook of Psychiatry, Oxford University Press, 2012, Lithium: 1192-1203

4. Sadock B.J., Sadock V.A. - Pocket Handbook of Psychiatric Drug Treatment, Lippincott Williams \& Wilkins, New York, 2011, 136-47

5. Dallocchio C., Mazzarello P. A case of Parkinsonism due to lithium intoxication: treatment with Pramipexole, J Clin Neurosci. 2002 May; 9(3):310-1. doi: http://dx.doi.org/10.1054/jocn.2001.1033

6. Cerqueira, Ana Claudia Rodrigues de, Reis, Moema Costa dos, Novis, Fernanda Demôro, Bezerra, José Marcelo Ferreira, Magalhães, Gérson Canedo de, Rozenthal, Márcia, \& Nardi, Antônio Egídio - Cerebellar degeneration secondary to acute lithium carbonate intoxication. Arquivos de Neuro-Psiquiatria, 66(3a), 578-580, 2008, https://dx.doi.org/10.1590/S0004$282 \times 2008000400032$

7. Sylvester R.K., Leitch J., Granum C. Does Acyclovir Increase Serum Lithium Levels?. Pharmacotherapy: The Journal of Human anism of intoxication syndrome. Secondary, the excretion of Lithium decreased and the low serum volume led to high blood concentration of Lithium. Later on, the gastrointestinal impairment which appeared slowly added to the vicious circle which involved hydration, excretion and serum concentration of Lithium producing and intoxication syndrome.

Therefore, though rarely found in infectious clinics, the intoxication syndrome is mandatory to be imediately diagnosed and treated if the clinical aspect and medical history raise any signs of Lithium intoxication.

Conflict of interest: none declared Financial support: none declared

Pharmacology and Drug Therapy, 16: 466-468, 1996, doi:10.1002/j.1875-9114.1996.tb02980.x

8. Toronjadze T., Polena S., Santucci T. Jr., Naik S., Watson C., Lakovou C., Babury M.A., Gintautas J. Prolonged requirement for ventilatory support in a patient with Eskalith overdose, Proc West Pharmacol Soc. 2005; 48:148-9.

9. Roy M., Stip E., Black D., Lew V., Langlois R. Cerebellar degeneration following acute lithium intoxication, Rev Neurol (Paris). 1998 Jul; 154(6-7):546-8.

10. Delva N.J., Hawken E.R. Preventing lithium intoxication. Guide for physicians. Can Fam Physician. 2001 Aug; 47:1595-600.

11. Chen K..P, Shen W.W., Lu M.L. Implication of serum concentration monitoring in patients with lithium intoxication. Psychiatry and Clinical Neurosciences, 58: 25-29, 2004, doi:10.1111/j.1440-1819.2004.01188.x

12. Rätz Bravo A.E., Egger S.S., Crespo S., Probst W.L., Krähenbühl S. Lithium intoxication as a result of an interaction with rofecoxib, Ann Pharmacother. Vol 38, Issue 7-8, pp. 1189-1193. Epub 2004 Jun 8. DOI: 10.1345/aph.1E034 